\title{
Les enjeux de la décentralisation
}

Le financement de l'éducation au Pakistan

The challenges of decentralisation. Funding education in Pakistan

La financiación de la educación en Pakistán y las dificultades frente a la

descentralización

\section{Nadeem Kayani}

Traducteur : Robert Elbaz, Sylvaine Herold et Marie-José Sanselme

\section{OpenEdition}

\section{Journals}

Édition électronique

URL : http://journals.openedition.org/ries/3695

DOI : 10.4000/ries.3695

ISSN : 2261-4265

\section{Éditeur}

Centre international d'études pédagogiques

\section{Édition imprimée}

Date de publication : 15 avril 2014

Pagination : 99-109

ISBN : 978-2-85420-603-6

ISSN : 1254-4590

Référence électronique

Nadeem Kayani, «Les enjeux de la décentralisation », Revue internationale d'éducation de Sèvres [En ligne], 65 | avril 2014, mis en ligne le 15 avril 2016, consulté le 06 janvier 2020. URL : http:// journals.openedition.org/ries/3695 ; DOI : 10.4000/ries.3695 


\section{Les enjeux de la décentralisation}

\section{Le financement de l'éducation au Pakistan*}

\section{Nadeem Kayani}

L'éducation est généralement perçue comme une expérience enrichissante pour l'individu, qui le transforme en citoyen et le dote des valeurs et des compétences sociales requises pour mener une vie meilleure. Loin de se limiter aux individus, cette optimisation des capacités ${ }^{1}$ concerne également les sociétés, qui en bénéficient et deviennent des espaces de développement holistique. Cela n'améliore pas seulement l'économie mais également les résultats en éducation.

Dans les pays en voie de développement comme le Pakistan, les politiques éducatives se sont concentrées sur des améliorations quantitatives, sans mettre en œuvre les aspects qualitatifs pourtant mentionnés dans les documents officiels. Conjugué à la pauvreté, l'accroissement de la population, principalement chez les jeunes, constitue aujourd'hui un défi pour rattraper le retard considérable pris dans la scolarisation des enfants et dans la lutte contre les inégalités en matière d'accès des filles à l'éducation, essentiellement dans les zones sous-développées. Ces problèmes liés à la qualité aboutissent à un taux très élevé d'abandon durant les toutes premières années de la scolarité. Les déficits budgétaires au Pakistan ont créé un contexte de ressources limitées, si bien que le système éducatif dépend du financement de donateurs étrangers. Ainsi, il semble que le Pakistan ne respectera pas l'échéance de 2015 pour l'atteinte de la plupart des Objectifs de développement du millénaire.

Cet article tente de répondre aux questions suivantes: le budget de l'éducation a-t-il été une priorité politique? Les évolutions législatives et les réformes récentes peuvent-elles conduire à une collaboration durable publicprivé au service du développement éducatif ? L'optimisation des capacités et des compétences (empowerment) au service des décisions administratives et financières au niveau local peut-elle aboutir à un meilleur service éducatif ? La participation de la communauté à travers les comités de gestion scolaire aboutit-elle à des résultats éducatifs tangibles?

\section{LA POLITIQUe ÉdUCATIVE}

Rédigée en 1973, la Constitution du Pakistan a prévu un enseignement gratuit et obligatoire d'une durée minimum avec un accès à l'enseignement technique et supérieur. Cet engagement nécessitait un effort de l'État dans la

\footnotetext{
* Article traduit par Robert Elbaz et Marie-José Sanselme.
}

1. Empowerment dans le texte (NdIR). 
durée, un engagement politique constant et l'allocation de ressources financières conséquentes; mais en fait l'éducation n'a jamais été une priorité politique, reléguée au second plan par la course au pouvoir des civils comme des militaires, qui monopolisait toute la vie nationale.

La Politique d'éducation nationale (National Education Policy) présentée en 2009 instaurait un lien positif entre éducation et développement, sans laisser place à des tendances clivantes, qui peuvent conduire à une régression économique et à une instabilité sociale. Cette politique mettait l'accent sur la montée en puissance des inscriptions dans les écoles du secteur public et sur un financement accru de l'éducation. Elle insistait sur la nécessité de réduire les déséquilibres entre zones urbaines et zones rurales ainsi qu'entre les genres, d'améliorer la qualité pédagogique à tous les niveaux, en particulier à travers la réforme du curriculum, de renforcer les infrastructures éducatives et d'encourager la participation du secteur privé et l'engagement effectif des communautés. Elle cherchait tout particulièrement à résoudre le problème des enfants non scolarisés ainsi qu'à favoriser l'émergence d'une éducation non formelle venant compléter le système formel.

Voté à l'unanimité par le Parlement en 2010, le $18^{\mathrm{e}}$ amendement représente un tournant historique dans l'histoire constitutionnelle du Pakistan. Le gouvernement fédéral a été déchargé de la gestion de dix-sept départements ministériels, dont celui de l'éducation, sans compter l'attribution aux gouvernements des provinces du pouvoir de prélever des impôts locaux, comme les taxes sur les ventes. Cet amendement mettait fin à la « liste simultanée ${ }^{2}$ » et conférait davantage d'autonomie aux provinces en matière d'éducation et de santé. Auparavant, la Commission nationale des Finances ${ }^{3}$, chargée de répartir les ressources entre les différents niveaux de gouvernement, avait accru la part des provinces dans la dotation budgétaire, pour accompagner les changements législatifs mentionnés ci-dessus.

Bien que, par le passé, l'éducation ait essentiellement relevé des compétences des provinces, le ministère fédéral de l'éducation jouait non seulement un rôle de coordination mais il définissait également les grandes lignes de la politique éducative et déterminait les curricula. En application du récent $18^{\mathrm{e}}$ amendement, les prérogatives du ministère de l'éducation ont été transférées aux provinces. Par là-même, cet amendement a eu de profondes implications sur la fourniture de services éducatifs.

Dans la phase initiale, on s'attend à ce que certains problèmes d'ajustement posent inévitablement un défi majeur aux provinces, étant donné leur

2. NdT : La « Liste législative simultanée » (Concurrent Legislative List) confère des pouvoirs législatifs aux gouvernements fédéral et provinciaux. Une fois certains éléments supprimés des textes relevant du 18e amendement, le gouvernement fédéral ne sera plus en mesure de légiférer sur tout sujet actuellement mentionné dans la liste, ce pouvoir étant alors exclusivement dévolu aux provinces. (Source : Dawn.com)

3. The National Finance Commission Award (NdT). 
pouvoir limité et leur manque d'expérience, tout spécialement en matière de définition d'une politique, de planification et de gestion. Cela peut signifier que certains services éducatifs pourraient connaître à court terme des difficultés. Toutefois, ces mêmes provinces mettent peu à peu en place les compétences et les capacités nécessaires, si bien que ces services devraient s'améliorer à l'avenir.

Le Pakistan a signé des engagements internationaux relatifs au secteur éducatif, qui prévoyaient de parvenir à une éducation primaire universelle en 2015 et d'assurer un maintien de la scolarisation jusqu'en $5^{\mathrm{e}}$ année du primaire, de parvenir à la littéracie des adultes et à la parité hommes-femmes. Les indicateurs éducatifs du Pakistan montrent que seule la parité devrait être atteinte à l'horizon 2015. Atteindre les autres objectifs nécessiterait un engagement encore supérieur et des efforts intenses. La mise en place de l'éducation primaire universelle exigera des ressources budgétaires accrues, aussi bien dans l'éducation formelle que non formelle, avec la participation des secteurs public et privé, ainsi qu'une amélioration de la qualité éducative, grâce à l'implication active des communautés.

\section{LE SCÉNARIO ÉDUCATIF AU PAKISTAN}

Le système éducatif du Pakistan souffre d'inégalités inhérentes. D’une part, le taux net de scolarisation est de $57 \%$. Près de sept millions d'enfants ne sont pas scolarisés, que ce soit dû à des difficultés d'accès à l'école, à la pauvreté, à l'absence d'enseignants, à des préjugés liés au genre ou encore à l'indisponibilité ou au manque de formation des enseignants. Même pour les enfants inscrits à l'école, l'accès de la population rurale à une éducation de qualité demeure limité.

L'existence de filières éducatives parallèles dans le pays, avec un secteur public offrant un enseignement gratuit débouchant sur une qualification locale et un secteur privé, assurant un enseignement onéreux et offrant une qualification internationale, est un autre obstacle à l'uniformisation de la qualité de l'enseignement. Ces disparités, nourries par des différences dans la langue d'enseignement (l'ourdou pour les strates inférieures de la société, l'anglais pour les classes les plus aisées), accentuent encore les clivages. Plus tard, ces différences se retrouvent dans la vie professionnelle et cimentent encore davantage les divisions de classes, qui finissent par conduire à des conflits.

Le contexte général et l'ampleur du défi éducatif au Pakistan peuvent être mieux compris à la lumière des données statistiques de 2011-2012, selon lesquelles le nombre total d'élèves scolarisés était de 40,1 millions d'élèves environ, étudiant dans 2,31 millions d'écoles et d'établissements, dans lesquels exerçaient 1,44 million d'enseignants. Comparé aux chiffres de 2010-2011, cela correspondait à une augmentation des inscriptions de 4,2\%, de 1,7\% du nombre d'établissements et de 2,1\% de celui des enseignants. Au Pakistan, le 
secteur non formel est réduit puisque seuls 0,25 million d'élèves ou d'étudiants sont inscrits dans 32500 établissements religieux, écoles de base non formelles, établissements techniques ou de formation des maîtres (Economic Survey of Pakistan, 2012-2013).

Les disparités dans l'offre éducative peuvent être observées entre différents niveaux de revenu, entre ruraux et urbains et entre genres. Les disparités entre zones rurales et urbaines sont flagrantes : $73 \%$ des citadins ont suivi une scolarité, contre seulement $53 \%$ des ruraux. Avec les inégalités dues au genre (les garçons sont plus scolarisés que les filles), la pauvreté joue un rôle essentiel dans les problèmes d'accès à l'éducation, mais cette situation évolue. Les disparités dues au genre diminuent mais les facteurs économiques demeurent cruciaux, tout particulièrement en matière d'accès à une éducation de qualité.

$\mathrm{Au}$ Pakistan, l'enseignement supérieur a connu une poussée récente grâce à la mise en place d'une Commission de l'enseignement supérieur. La faiblesse des standards éducatifs, le faible pourcentage d'inscriptions, le manque de financement et de pertinence dans la recherche font que l'enseignement supérieur contribue très peu au développement national. Les universités sont contrôlées par la commission, alors que les collèges universitaires dépendent des gouvernements des provinces.

L'enseignement privé est devenu un acteur important de la scène éducative pakistanaise. Ce contexte particulier apparait dans les données disponibles pour l'année 2007-2008. Il représente $30 \%$ du nombre total d'établissements, $44 \%$ des enseignants travaillant dans le pays et $34 \%$ du nombre total d'inscriptions. Il n'est pas facile d'évaluer la qualité de l'enseignement dispensé par le privé, comparé au public. Les écoles privées bénéficient d'un a priori positif mais il existe aussi de très bonnes écoles publiques. À l'avenir, la mise en place d'une réglementation du secteur privé égalisera les conditions de la concurrence entre les secteurs. La croissance du secteur privé pakistanais offre un moyen de répondre aux défis que posent l'inégalité d'accès à l'école, les disparités dues au genre et l'accès à une éducation de qualité dans un environnement économique défavorable.

\section{LES RESSOURCES POUR L'ÉDUCATION}

Le défi posé par les problèmes de quantité et de qualité au Pakistan exige de donner la priorité au budget de l'éducation. Les taux de décrochage scolaire sont élevés et un grand nombre d'enfants ne sont pas scolarisés. Cette situation suppose la mise en place rapide d'une meilleure formation des enseignants, de nouvelles infrastructures, de manuels scolaires aisément compréhensibles et de méthodes d'évaluation qui ne reposent pas uniquement sur le système actuellement en vigueur, fondé sur la réussite et sur l'échec. Cet investissement devra être conduit à la fois par les secteurs public et privé. Outre cet 
investissement dans la scolarisation formelle, il faudra également accroître les possibilités d'accès à l'enseignement non formel, en collaboration avec le secteur privé.

Si l'on excepte le Bangladesh, les crédits destinés à l'éducation au Pakistan sont les plus faibles de la région en termes de pourcentage du PIB, aux environs de $2 \%$ durant la dernière décennie. Les dépenses réelles sont même inférieures à $1,5 \%$, les prévisions budgétaires n'étant pas toutes exécutées. L'essentiel du budget va à l'enseignement primaire, puis au secondaire et enfin à l'enseignement supérieur.

Deux études, qui analysent les efforts budgétaires nécessaires pour parvenir aux objectifs fixés, montrent qu'atteindre un taux net de scolarisation de $100 \%$ en primaire à l'horizon 2015/2016 exigerait, outre des améliorations massives en matière de gouvernance et de mise en œuvre, une enveloppe de quelque 1300 milliards de roupies, soit 9,1 milliards d'euro ${ }^{4}$. Faire accéder 36 millions de personnes à la littéracie dans les cinq prochaines années coûterait 178 milliards de roupies (1,2 milliards d'euro). Une autre étude menée afin d'évaluer les ressources nécessaires pour atteindre l'objectif d'éducation primaire universelle en 2015/2016 souligne que le Pakistan doit de toute urgence investir massivement dans l'enseignement primaire. Pour maintenir la tendance actuelle de $30 \%$ de scolarisation dans le privé, le secteur public doit prendre en charge les $70 \%$ restants, tout en tenant compte du contexte actuel d'inflation galopante et de hausse des salaires (UNESCO, 2012).

L'éducation est considérée comme un bien public. Le rôle du secteur public est donc vital dans l'offre éducative et pour l'accès à l'éducation de chaque strate de la société. Dans l'ensemble, la plupart des ressources destinées à l'éducation proviennent du secteur public. Elles représentent environ $80 \%$ des dépenses nationales totales, alors que $20 \%$ proviennent du secteur privé. Au Pakistan, les dépenses d'éducation représentent $4 \%$ des ressources publiques, soit 2 à $3 \%$ du budget fédéral et entre 6 et $10 \%$ du budget des provinces.

La défaillance du secteur public fait que les dépenses prévues au budget ne sont pas totalement réalisées. Les dépenses réelles ont récemment augmenté dans les provinces les plus petites. Les dépenses de fonctionnement représentent 80 à $90 \%$ des dépenses totales, alors que le sous-secteur du primaire, en baisse, en absorbe de 38 à $48 \%$. Quant à l'enseignement supérieur, qui dépend toujours du gouvernement fédéral, il représente entre 16 et $26 \%$ des dépenses totales et sa part ne cesse d'augmenter (UNESCO, 2011).

En outre, l'efficacité de ces dépenses est encore réduite en raison de problèmes dans les structures de gouvernance elles-mêmes, au niveau fédéral, comme à celui des provinces ou des districts. Les transferts à partir du budget du gouvernement fédéral se font sous forme de dotation globale attribuée aux 
provinces par la Commission nationale des finances. Le critère retenu est la population, et les décisions concernant les dépenses sont déconnectées des ressources fiscales, tant au niveau des provinces qu'à celui de l'État fédéral. Ces transferts ont contribué à financer la plupart des dépenses dans les provinces les plus petites.

Au Pakistan, la défense et le service de la dette sont les postes budgétaires prioritaires, non seulement dans les budgets votés mais encore davantage dans les budgets réalisés, les dépenses étant souvent supérieures aux crédits votés. Cette situation reflète le fait que l'on n'accorde pas à l'éducation la place qui lui est due. La situation géopolitique du Pakistan et les erreurs de gestion sont responsables de l'inadaptation du budget et de la diminution constante des dépenses réellement engagées en matière d'éducation, alors même que les budgets nécessaires au développement ne cessent d'être réduits en ces temps de difficultés économiques.

Au Pendjab, l'une des initiatives récentes a été d'augmenter l'efficacité des dépenses éducatives en alignant les crédits alloués aux écoles sur le coût par élève, mais cela risque de promouvoir davantage les établissements déjà développés en matière d'infrastructures, aux dépens des écoles situées dans des zones sous-développées. Il semble toutefois nécessaire d'établir la dotation budgétaire des établissements en fonction des dépenses par élève, processus que l'on pourra au bout du compte lier à l'obtention de meilleurs résultats. Cela peut favoriser l'efficience des dépenses du secteur public en matière éducative.

$\mathrm{Au}$ Pakistan, les financements de l'aide internationale complètent les dotations budgétaires du secteur public dans le domaine de l'éducation. Il a été difficile de parvenir à ce résultat car les politiques de financement des donateurs, au vu de la situation géopolitique, ont reflété en priorité leur agenda politique propre plutôt que les nécessités du développement de l'éducation du pays. Les donateurs ont fait évoluer les modalités de leur aide, passant de prêts conditionnels à un soutien budgétaire direct. Aujourd'hui, une approche sectorielle est privilégiée. L'aide bilatérale se présente la plupart du temps sous la forme de dons, alors que l'aide multilatérale est essentiellement constituée de prêts.

Comme le soutien budgétaire est devenu le mode d'action préférée des donateurs internationaux les plus actifs, on ne peut plus distinguer l'impact du financement public de celui de l'aide (Malik, R. et Naveed, A., 2012). Bien que le Pakistan ait moins dépendu de l'aide extérieure que les autres pays en voie de développement de la région, les financements apportés par les donateurs en matière d'éducation ont contribué à l'amélioration des résultats éducatifs du pays mais il subsiste des disparités significatives entre les sexes, les régions, les villes et les campagnes. Ces contributions ont drainé des fonds vers les zones économiquement défavorisées du pays, ou dans les domaines les moins prioritaires en matière d'éducation, essentiellement l'enseignement primaire et la formation des maîtres. 
Le secteur privé, qui était essentiellement urbain et florissant, a été absorbé par l'État durant la période de nationalisation de l'industrie dans les années 1970. Cette position a été ultérieurement infléchie lorsque l'on a remis en cause la qualité de l'enseignement dans les établissements publics et que l'on a repris les investissements dans ce secteur.

Le secteur privé a depuis enregistré une croissance rapide, pour devenir un prestataire essentiel des services éducatifs au Pakistan, à la fois en termes absolus et par rapport au secteur public. Si l'on excepte les établissements privés de prestige pour la population la plus fortunée, les établissements privés les moins chers fournissent une solution qui permet de répondre au défi quantitatif, c'est-à-dire aux objectifs de scolarisation et aux problèmes d'infrastructures. Si le secteur public demeure le dernier recours de la classe la plus défavorisée et de la classe moyenne, les établissements privés peuvent contribuer au développement éducatif du Pakistan.

Entreprise en 1999-2000, la seule enquête relative au secteur privé de l'enseignement faisait apparaître un investissement annuel de 2,5 milliards de roupies (17,5 millions d'euro) et une enveloppe salariale de 12 milliards de roupies (84 millions d'euro). Le retour sur investissement net du secteur privé s'élevait à $52 \%$ de la dépense d'investissement et de fontionnement. Ce taux montre tout l'intérêt pour les acteurs économiques cherchant à faire fructifier leur capital d'investir dans le secteur privé d'éducation, ce qui pourrait à terme conduire à une expansion rapide du secteur (I-SAPS, 2010). Ainsi, les services éducatifs du secteur privé, intervenant en complément du service public et travaillant de concert avec lui, peuvent assurer la scolarisation des élèves et fournir une éducation de qualité, sachant que le financement nécessaire pour atteindre les objectifs nationaux et internationaux ne peut être assuré sur les seuls deniers publics.

\section{UN PARTENARIAT PUBLIC-PRIVÉ}

Il y a longtemps que l'idée de partenariats public -privé (PPP) a fait son chemin au Pakistan et qu'elle a été mise en pratique. Le développement le plus significatif dans ce domaine a eu lieu au début des années 1990, avec la création de fondations pour l'éducation à l'échelle des provinces et du pays. La modalité la plus fréquemment utilisée par ces fondations est la combinaison d'un financement public et de fonds privés. En conséquence, les dotations financières des gouvernements fédéral et provinciaux à ces fondations pour la promotion de ces partenariats ont augmenté de façon significative au cours des dernières années. En 2009-2010, le gouvernement du Pendjab a alloué 4 milliards de roupies (28 millions d'euro) dans ce but. Au total, 4,9 milliards de roupies (34,3 millions d'euro) ont été consacrés à ces fondations en 2009-2010, en augmentation de $28 \%$ par rapport à 2007-2008. 
Une question clef, en ce qui concerne le rôle des PPP dans le secteur éducatif, est l'absence de cadre politique harmonisé. Les gouvernements fédéral et provinciaux expérimentent différents dispositifs de mise en œuvre. De la même façon, il n'existe pas de cadre uniforme permettant de s'assurer de la qualité des écoles privées résultant d'un partenariat avec le gouvernement. Même si des mesures adéquates sont prises pour assurer cette qualité, le potentiel nécessaire pour faire monter en puissance des modèles de PPP ayant fait leurs preuves demeure limité, en raison des contraintes budgétaires, étant donné que presque tous les partenariats reposent sur un schéma de financement public associé à des fonds privés. Afin de tirer le meilleur parti de ce mécanisme, le gouvernement doit garantir les ressources financières nécessaires à cette fourniture de services éducatifs grâce aux PPP.

\section{Gouvernance/DÉvolution EN ÉDUCATION}

Le concept de décentralisation ou de gouvernement décentralisé implique la répartition de l'autorité entre les institutions de gouvernance, aux niveaux central, régional et local. On met donc implicitement l'accent sur une responsabilité conjointe pour résoudre les problèmes, en vue d'obtenir à terme un meilleur service. La dévolution suppose le transfert du pouvoir et des ressources à une institution locale démocratique et indépendante. De par sa nature même, la décentralisation maintient la stabilité politique du système et aboutit à l'amélioration du service dans les secteurs sociaux ciblés au niveau local.

L'expérience pakistanaise du transfert du pouvoir au niveau local a été historiquement mise en œuvre par les régimes militaires en quête de légitimité. Dans les périodes de gouvernement démocratique, cette politique s'est toujours heurtée aux administrations provinciales, qui ne désiraient pas déléguer leur pouvoir à des instances de gouvernance subalternes. Historiquement, la gouvernance au niveau local a été contrôlée par le gouvernement fédéral en contournant les gouvernements des provinces. Grâce aux changements législatifs et aux transferts financiers réalisés depuis le niveau fédéral vers les gouvernements provinciaux, ceux-ci se voient dotés d'une réelle capacité de gouvernance et des ressources financières nécessaires pour offrir un service effectif au niveau local. Le risque principal est la possibilité de mainmise des élites locales sur cette dévolution, ainsi que le risque de court-circuit des représentants élus au niveau local, comme on l'a vu par le passé.

Le transfert des pouvoirs dans le cadre de la dévolution comprend la délégation de l'autorité sur les problèmes financiers, administratifs et éducatifs. Ce schéma de gouvernance a un impact durable sur la prestation de services dans les secteurs sociaux, et particulièrement en matière éducative (Shah D., 2003). Les responsabilités des provinces en matière de recrutement du personnel, de transferts à l'intérieur du district et de paiement des salaires, au moyen 
éventuellement des fonds des projets de développement, sont confiées aux décisionnaires locaux. Dans le contexte pakistanais, cette expérience de décision décentralisée conduira à une amélioration de la prestation des services sociaux, si le transfert du pouvoir et des ressources depuis les provinces vers les districts est effectué (Paracha, Saad, Abdullah, 2033).

\section{LES FLUX FINANCIERS \\ DANS LE CADRE DE LA DÉVOLUTION}

$\mathrm{Au}$ Pakistan, le gouvernement fédéral a toujours eu un rôle dominant dans le contrôle des recettes fiscales. Après 2001, durant la mise en place du système de gouvernement local, le gouvernement fédéral a continué à percevoir les impôts sur les revenus, les propriétés, les biens et les services, alors que le gouvernement provincial gardait la collecte des impôts agricoles, fonciers et indirects. De nouveaux impôts, comme la taxe professionnelle, conçus à l'origine pour être collectés par le gouvernement de district, n'ont pas été mis en place et l'essentiel du pouvoir de lever l'impôt est resté aux instances gouvernementales, fédérale et provinciales. La capacité d'action des gouvernements locaux a été d'autant plus réduite que la taxe de passage, une source essentielle de leurs revenus, a été supprimée.

Les capacités budgétaires des gouvernements locaux ont significativement augmenté sous les régimes militaires et diminué sous les régimes démocratiques, ce qui a abouti à un affaiblissement de la gouvernance locale au Pakistan. Ce contraste peut s'expliquer par le fait que les premiers ont eu tendance à encourager un semblant d'autonomie locale, afin de rechercher une certaine légitimité, et à court-circuiter les partis politiques, alors que les seconds, au niveau central comme dans les provinces, ont perçu les gouvernements locaux comme des concurrents capables de réduire leur influence sur la vie des gens (Shah, Anwar, 2012).

Les possibilités d'emprunt des provinces ont été étendues aux prêts intérieurs et étrangers, dans certaines limites et à certaines conditions. Le $18^{\mathrm{e}}$ amendement a conduit à un changement de scénario, dans lequel les gouvernements des provinces se sont vu confier le pouvoir de prélever l'impôt sur les ventes et sur la propriété, mais d'autres transferts en direction des districts, sur des critères de niveau de revenu, de densité de population et d'effort fiscal, auront pour conséquence une offre éducative efficiente au niveau local.

\section{Participation DE LA COMMUNAUTÉ}

De manière générale, la participation des communautés au processus local de décision a fait son chemin. La responsabilisation des élus locaux en 
matière de gestion des établissements d'enseignement locaux donne l'occasion de renforcer ces derniers et de fournir des services avec efficience. La dévolution insiste sur l'autonomie des établissements, car l'autorité centrale ne peut réagir de façon appropriée aux besoins changeants des communautés.

Ce qui manque de façon cruciale pour accompagner cette réforme et l'inscrire dans le temps, c'est une stratégie destinée à identifier les ressources humaines ayant le potentiel de la conduire plus avant. En effet, c'est seulement lorsqu'existe une combinaison de leadership et d'encouragement institutionnel que les établissements semblent susceptibles de parvenir à un certain niveau de qualité. Ils se concentrent sur l'identification et la promotion de ressources humaines prometteuses dont ils disposent et mettent en place un système logique et cohérent de valorisation des enseignants et des directeurs d'établissements propre à améliorer la qualité des écoles.

Les conseils d'établissement, cette institution essentielle au niveau le plus local, n'ont pas été un succès, dans la mesure où ils comprenaient principalement des enseignants à la retraite et où leur pouvoir de décision se limitait à des dépenses mineures liées à l'infrastructure. Ces conseils d'établissement doivent être directement financés et responsabilisés, afin d'uniformiser le cadre légal dans lequel les décisions sont prises. On peut considérer l'initiative récente destinée à mettre en place des autorités éducatives de district au Pendjab comme une tentative de gérer le système éducatif à ce niveau mais, sans mise en place d'une dévolution locale, il sera difficile d'étendre cette initiative d'un gouvernement provincial.

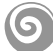

La fourniture de services éducatifs dépend sans aucun doute des enveloppes budgétaires mais la démocratisation des structures de gouvernance fournit l'occasion de mettre les schémas de financement en conformité avec les priorités en matière d'éducation, afin de soutenir la population la plus jeune, ouvrant ainsi la voie au développement économique. L'accès à l'éducation, dans un pays en voie de développement comme le Pakistan, demeurera un enjeu capital et le rôle du gouvernement ne doit pas être sous-estimé. Les initiatives publiques et privées, comme les fondations éducatives destinées à financer l'éducation des classes les plus défavorisées, doivent être pérennisées.

Le Pakistan a fait l'expérience à la fois de la décentralisation et de la dévolution, mais les élites politiques locales, crispées sur leurs privilèges, ont détourné le système à leur profit, tout particulièrement dans les régions rurales. La création des comités de gestion scolaire est une occasion de donner la capacité aux communautés locales de participer aux décisions, non seulement pour développer les infrastructures, mais également pour obtenir une éducation de qualité. 


\section{Bibliographie}

MALIK R. \& NAVEED Arif (2012) : Financing education in Pakistan: The impact of public expenditure and aid on educational outcomes, RECOUP working paper $n^{\circ} 42$. 39 pages téléchargeables sur : http://goo.gl/hO0UHd/

Government of Pakistan, Ministry of Finance (2013) : Economic survey of Pakistan, Islamabad: Government of Pakistan, Ministry of Finance.

I-SAPS (2010) : "Private Sector Education in Pakistan: Muse or Musing", Islamabad: Institute of social policy sciences.

JAMIL, BAELA \& RAZA (2002): Decentralization and Devolution: Educational implications of the praetorian interpretation, Lahore: Idara-e-Taleem-o-Aagahi Public Trust.

PARACHA, SAAD \& ABDULLAH (2003): Devolution plan in Pakistan, context, implementation and issues. Open Society Institute, Budapest, Hungary.

PILDAT (2010) : "Financing quality basic education for all in Pakistan". Briefing papers $\mathrm{n}^{\circ} 38$, Islamabad: Pakistan institute of legislative development and transparency.

SHAH Anwar (2012) : The $18^{\text {th }}$ constitutional Amendment: Glue or solvent for nation building and citizenship for Pakistan? The Lahore Journal of Economics, 17: SE, p. 384-424.

SHAH Dawood (2003) : Country Report on Decentralization of education in Pakistan: Policies and strategies Decentralization in the education system in Pakistan: Policies and strategies, Islamabad: Academy for Educational Planning and Management.

UNESCO (2011) : Macro-trends for financing of Education in Pakistan, Islamabad: UNESCO.

UNESCO (2012) : Situational analysis of Education sector in Pakistan, Islamabad: UNESCO. 
